\title{
IORT with mobile linacs: the Italian experience
}

G. Tosi, M. Ciocca

\section{Introduction}

At the beginning of 1999 a mobile Linac (Novac 7, manufactured by Hitesys, Aprilia, Italy) able to produce electron beams in the nominal energy range 4.5-9 MeV became available at the European Institute of Oncology, Milano, Italy for being employed in "traditional" operating rooms, especially for the IORT of early breast cancer patients, alternative to conventional postsurgical radiotherapy. Until april $30^{\text {th }} 2004,899$ early breast cancer patients and 25 patients with other type of tumours have been treated wilh this linac, without any type of accident. Due to the increase of the demand of this type of radiation treatment, at the beginning of February 2004 a second unit came into operation (LIAC manufactured by Info \&Tech, Roma) able to produce electron beams in the nominal energy range 4-10 $\mathrm{MeV}$. In this paper, the main physical and dosimetrical problems related with the clinical use of these linacs will be treated.

\section{Mechanical and physical characteristics of mobile linacs}

Both Novac 7 and LIAC are specifically designed for being employed in a conventional operating room (s. Fig. 1). They are mounted in a motorised structure that can be easily displaced inside the operating room thanks to a caster installed in the base, and pushed very slowly towards the operating table and the patient, so as to be easily positioned with great accuracy in the treatment position.

Novac 7 is equipped with three sets of four plexiglas applicators each, plane $\left(0^{\circ}\right)$ and bevelled $\left(22.5^{\circ}\right.$ and $\left.45^{\circ}\right)$, whose characteristics are shown in Table I and Fig. 2. The available applicators of LIAC are listed in Table II.
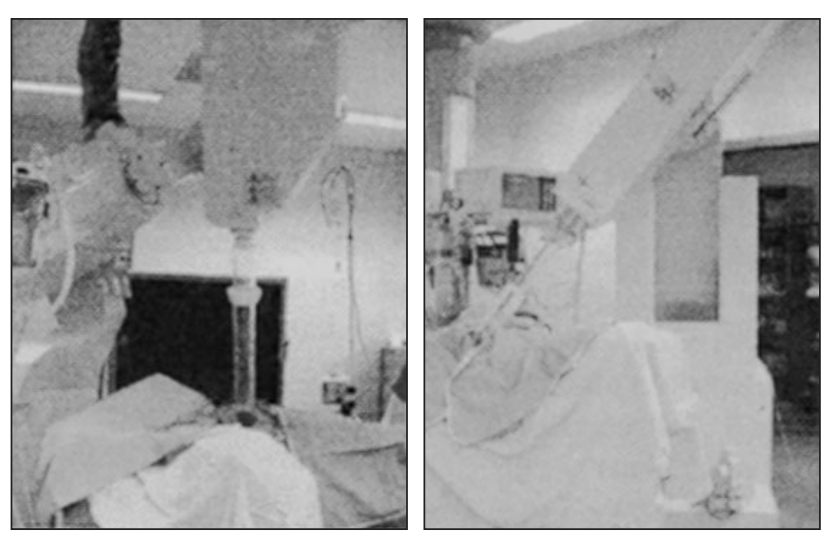

Fig. 1. The mobile linacs Novac 7 and LI4C with an applicator mounted on the radiating head ready for the treatment in the operating room.

TABLE I

\begin{tabular}{lcc}
\hline \multicolumn{3}{c}{ Available applicators for Novac 7} \\
\hline Applicator's diameter $(\mathrm{cm})$ & SSD $(\mathrm{cm})$ & Bevel angles \\
\hline 4 & 80 & $0^{\circ}-225^{\circ}-45^{\circ}$ \\
5 & 80 & $0^{\circ}-225^{\circ}-45^{\circ}$ \\
6 & 80 & $0^{\circ}-22.5^{\circ}-45^{\circ}$ \\
8 & 80 & $0^{\circ} 22.5^{\circ}-45^{\circ}$ \\
10 & 100 & $0^{\circ}-22.5^{\circ} 45^{\circ}$ \\
\hline
\end{tabular}

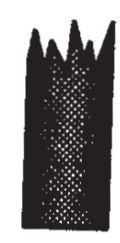

$\left(0^{\circ}\right)$

$\left(45^{\circ}\right)$

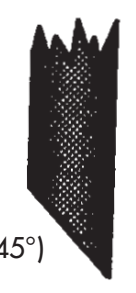

Fig. 2. Plane $\left(0^{\circ}\right)$ and bevelled $\left(45^{\circ}\right)$ applicators.

European Institute of Oncology Milano (Italy) 
TABLE II

\begin{tabular}{lcc}
\hline \multicolumn{4}{c}{ Available applicators for LIAC } \\
\hline Applicator's diameter $(\mathbf{c m})$ & SSD (cm) & Bevel angles \\
\hline 3 & 60 & $0^{\circ}-15^{\circ}-30^{\circ}-45^{\circ}$ \\
4 & 60 & $0^{\circ}-15^{\circ}-30^{\circ}-45^{\circ}$ \\
5 & 50 & $0^{\circ}-15^{\circ}-30^{\circ}-45^{\circ}$ \\
6 & 60 & $0^{\circ}-15^{\circ}-30^{\circ}-45^{\circ}$ \\
7 & 60 & $0^{\circ}-15^{\circ}-30^{\circ}-45^{\circ}$ \\
8 & 60 & $0^{\circ}-15^{\circ}-30^{\circ}-45^{\circ}$ \\
10 & 60 & $0^{\circ}-15^{\circ}-30^{\circ}-45^{\circ}$ \\
12 & 60 & $0^{\circ}-15^{\circ}-30^{\circ}-45^{\circ}$ \\
\hline
\end{tabular}

According to the manufacturer, Novac 7 can deliver electron beams of 4 nominal energies: $3,5,7$ and $9 \mathrm{MeV}$; the emission of electrons is pulsed, with a frequency of $5 \mathrm{~Hz}$, a pulse duration very short $(4 \mu \mathrm{s})$ and a very high dose/pulse $(1.9 \div 8.5 \mathrm{cGy} /$ pulse, depending on energy and applicator's diameter). In order to reduce the production of Bremsstrahlung, and consequently to reduce the radiation protection problems, no scattering foil is present aloog the path of the electrons from the exit window to the patient; the enlargement of the beam's section is due only to the geometrical divergence and to the rather large nominal SSD $180 \mathrm{~cm}$ for the applicators with a diameter $\leq 8 \mathrm{~cm}$ and $100 \mathrm{~cm}$ for the applicators with a diameter $=10 \mathrm{~cm}$ ). On the other side, LIAC can deliver electron beams of 4 nominal energies: $4,6,8$ and $10 \mathrm{MeV}$; the emission of electrons is pulsed, with a frequency of $10 \mathrm{~Hz}$ (in the clinical modality, $20 \mathrm{~Hz}$ in the physical modality), a short pulse duration $(10 \mu \mathrm{s})$ and a very high dose/pulse $(0.2 \div 4.5 \mathrm{cG} /$ pulse, depending on energy and applicator's diameter) and dose-rate (1.5-27 Gy/min, depending on energy). In order to reduce the length of the applicators, LIAC is equipped with a scattering foil $(80 \mu \mathrm{m}$ brass), that increases the amount of braking radiation. However, Bremsstrahlung is produced also as a consequence of the interaction of the electron beams with the patient's tissues. For this reason, before using both Novac 7 and LIAC in the operating rooms (placed at the second floor of the building) a very rigorous acceptance test was planned. For safety reason, all the evaluations and measurements were performed at the underground level of the building, inside a bunker where a $25 \mathrm{MeV}$ conventional linac was installed and operating. The main parameters checked both during the acceptance test and in the course of the quality control program are the following:

- User's interface

- Stability (constancy of the emission)

- Actual energy of the beams

- Beams profiles (symmetry and flatness)

- Isodose curves in water

- Dose (Gy/MU-Gy/pulse) and dose rate

- Stray radiation (leakage radiation from the radiating head-Bremsstrahlung- scattered radiation)

- Mechanical characteristics and reliability, connection to the earth, warnings, etc.

The evaluation of the actual energy $E_{p, 0}$ of the beams was based on the measurement in a water phantom and using

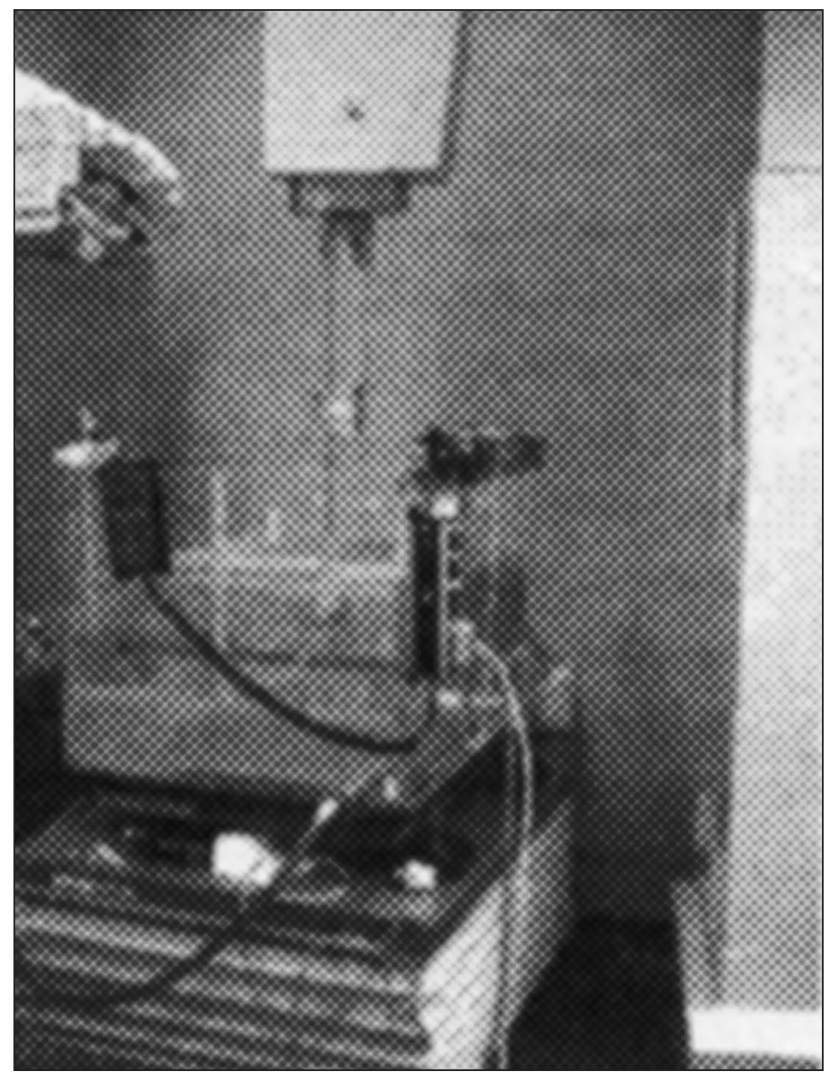

Fig. 3. Experimental set-up for the determination of the percentage depth dose curves (PDD), of the practical range $\left(R_{p}\right)$ and of the actual energy $\left(E_{p, 0}\right.$ of the electron beams.

small diodes (s. Fig. 3) of the depth dose curves along the beam's central axis, the determination of the practical range $R_{p}$ and the use of the following range-energy relationship:

$$
E_{p, 0}=C_{1}+C_{2} R_{p}+C_{3} R_{p}^{2}
$$

where:

$\mathrm{C}_{1}=0.22 \mathrm{MeV}$

$\mathrm{C}_{2}=1.98 \mathrm{MeV} \mathrm{cm}^{-1}$

$\mathrm{C}_{3}=0.0025 \mathrm{MeV} \mathrm{cm}^{-2}$

As shown in Table 3, the actual energies of the beams are significantly different than those declared by the manulactu-

TABLE III

Nominal and actual energies of the electron beams

Energies declared by the manufacturers and actual energies $E_{p, 0}$

\begin{tabular}{cccc}
\hline \multicolumn{2}{c}{ Novac 7 } & \multicolumn{2}{c}{ LIAC } \\
\hline $3 \mathrm{MeV}$ & $4.5 \mathrm{MeV}$ & $4 \mathrm{MeV}$ & $4.85 \mathrm{MeV}$ \\
$5 \mathrm{MeV}$ & $5.2 \mathrm{MeV}$ & $6 \mathrm{MeV}$ & $5.95 \mathrm{MeV}$ \\
$7 \mathrm{MeV}$ & $6.5 \mathrm{MeV}$ & $8 \mathrm{MeV}$ & $7.95 \mathrm{MeV}$ \\
$9 \mathrm{MeV}$ & $7.8 \mathrm{MeV}$ & $10 \mathrm{MeV}$ & $9.95 \mathrm{MeV}$ \\
\hline
\end{tabular}




\section{G. Tosi y M. Ciocca}

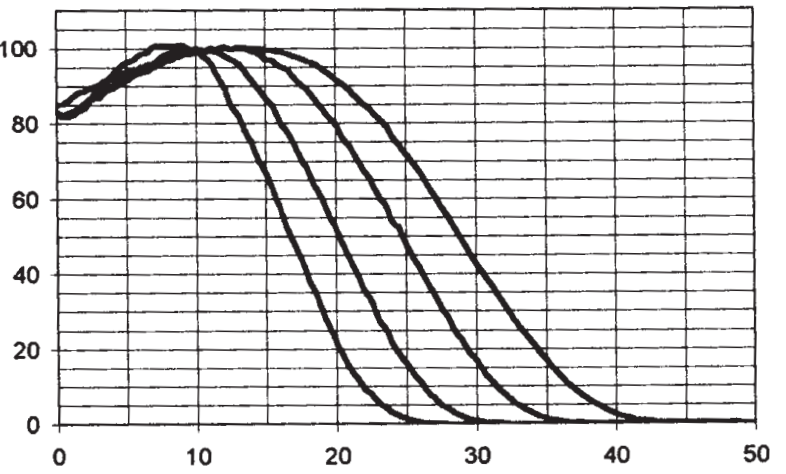

Fig. 4. Depth dose curves in water for Novac 7. From links to right, nominal energies of 3,5,7 and $9 \mathrm{MeV}$. In abscissa: depth in $\mathrm{mm}$ in ordinate: percentage depth dose, normalised to the maximum.

rer for Novac 7, and very near to the declared values for LIAC.

The depth dose curves for the electron beams generated by Novac 7 are illustrated in Fig. 4 .

In the following tables the depth of maximum and of $80 \%$ of the dose in water for plane applicators of Novac 7 and LIAC are reported.

TABLE IV

Depth of maximum and of $80 \%$ dose in water for Novac 7

\begin{tabular}{ccc}
\hline $\begin{array}{c}\text { Nominal energy } \\
(\mathbf{M e V})\end{array}$ & $\begin{array}{c}\text { Depth of maximum } \\
\text { dose }(\mathbf{m m})\end{array}$ & $\begin{array}{c}\text { Depth of } 80 \% \\
(\mathbf{m m})\end{array}$ \\
\hline 3 & 7 & 13 \\
5 & 10 & 15 \\
7 & 12 & 20 \\
9 & 15 & 24 \\
\hline
\end{tabular}

TABLE V

\begin{tabular}{ccc}
\hline Depth of maximum and of $\mathbf{8 0 \%}$ dose in water for LIAC \\
\hline $\begin{array}{c}\text { Nominal energy } \\
(\mathrm{MeV})\end{array}$ & $\begin{array}{c}\text { Depth of maximum } \\
\text { dose }(\mathrm{mm})\end{array}$ & $\begin{array}{c}\text { Depth of } 80 \% \\
(\mathbf{m m})\end{array}$ \\
\hline 4 & 8 & 13 \\
6 & 9 & 18 \\
8 & 12 & 23 \\
10 & 13 & 29 \\
\hline
\end{tabular}

For the absolute calibration of the dose, a set of Fricke dosimeters calibrated, delivered and evaluated at the ENEA Laboratories of Casaccia (Roma) were used. For the check of the dose that is performed in a solid phantom before each treatment, a flat ionisation chamber (Markus or Roos type) calibrated against the Fricke dosimeters and GafChromic films, calibrated in "conventional" electron beams are used.

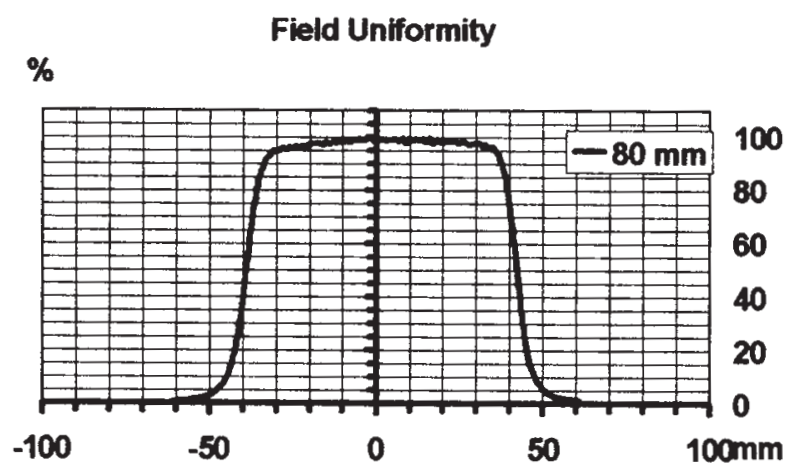

Fig. 5. Profile at the depth of maximum dose of an electron beam (nominal energy $9 \mathrm{MeV}$, plane applicator $\Phi 80 \mathrm{~mm}$ ) - Novac 7 .

The measurement of the beams profiles at the depth of maximum dose was made using small diodes; both the flatness and the symmetry are very good, as is shown in Fig. 5.

\section{Radiation protection problems}

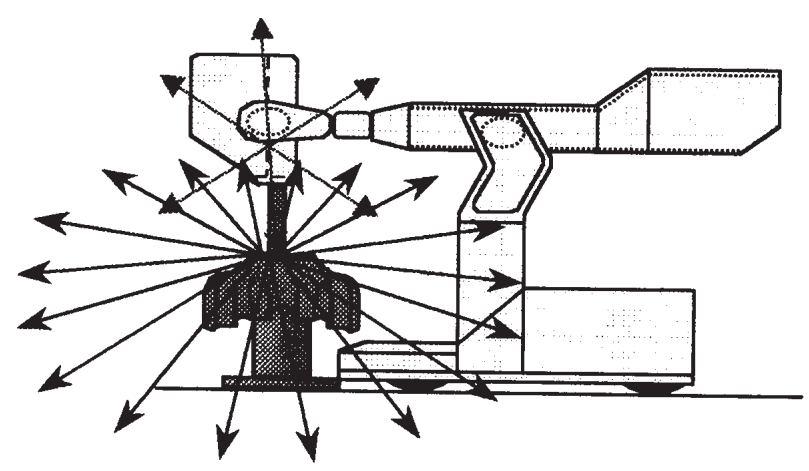

Fig. 6. Sources of stray radiation in the use of Novac 7 (a similar situation occurs for $(I A C)$.

The stray radiation generated by the two mobile linacs is made up by four different components (s. Fig. 6):

1. low energy radiation, scattered into the environment by the patient or by any object present along the beam's path.

2. leakage from the radiating head.

3. electrons emerging from the applicator's walls (PMMA, $5 \mathrm{~mm}$ thick) and scattered at various angles.

4. Bremsstrahlung generated by the interaction of the electron beam with the patient or any other object present along its path.

In order to evaluate both the intensity and the "quality" of such environmental radiation, some different detectors have been employed, namely:

- a ionization chamber Victoreen mod. 450 P.

- a radiation GM monitor Rados RDS-120 Universal Sur-

vey Meter, equipped also with a beta-ray probe.

- a radiation monitor (proportional counter) FAG Contamat FHT $111 \mathrm{M}$. 
The measurements of this stray radiation were made at the maximum nominal energies of the linacs $(9 \mathrm{MeV}$ and 10 $\mathrm{MeV}$ respectively), and irradiating a PMMA phantom $(30 \mathrm{~cm}$ $\times 30 \mathrm{~cm} \times 10 \mathrm{~cm}$ ) using the plane applicators having a diameter of $10 \mathrm{~cm}$ and working therefore at a SSD $=100 \mathrm{~cm}$ for Novac 7 and $60 \mathrm{~cm}$ for LIAC. Two main conclusion could immediately be drawn:

- the components 1. and 3. (low energy radiation and electrons emerging from the applicator's walls) can be completely absorbed by $5 \mathrm{~mm} \mathrm{~Pb}$.

- the intensity of the Bremsstrahlung strongly depends on the angle, being maximum in the direction of the beam (angle $=0^{\circ}$ ) and minimum in the opposite direction (angle $=180^{\circ}$ ), according to the second column of Table 7.

- also the energy, evaluated through the measurement of the attenuation in lead depends on the angle, as can be seen in the third column of the same table, where the TVL (Tenth Value Layers) are listed.

Moreover, in order to evaluate the attenuation produced by the floor and by the ceiling of the operating rooms $(25 \mathrm{~cm}$

TABLE VI

Angular dependence of the intensity of the Bremsstrahlung

\begin{tabular}{ccc}
\hline Angle & \% intensity & TVL (mm Pb) \\
\hline $0^{\circ}$ & 100 & 50 \\
$30^{\circ}$ & 14 & 45 \\
$45^{\circ}$ & 8 & 40 \\
$60^{\circ}$ & 3 & 35 \\
$90^{\circ}$ & 1.3 & 32 \\
$135^{\circ}$ & 0.2 & 30 \\
$180^{\circ}$ & 0.04 & 30 \\
\hline
\end{tabular}

concrete), the attenuation properties of concrete were evaluated, and TVL of $26 \mathrm{~cm}$ at $0^{\circ}$ and of $20 \mathrm{~cm}$ at $180^{\circ}$ were found.

In order to design the mobile shields to be used during the functioning of the linac in a conventional operating room, measurements of dose-rate in air at various distances and with different thickness of lead were pertormed. The results for Novac 7, expressed in $\mu \mathrm{Gy} / 100$ pulses are reported in Table VII.

The following working loads were estimated:

-400 treatments/operating room/year

- 30Gy/treatment

A dose constraint of $1 \mathrm{mSv} /$ year/operator (surgeons, anaesthetist, radiotherapist, medical physicist, etc.) was taken into account; the same dose constraint was established for the people living in the underlying and overlaying spaces. On the basis of the above mentioned evaluations, 4 mobile barriers mounted on casters and having the following characteristics were designed and built:

- height $: 150 \mathrm{~cm}$.

- width $: 120 \mathrm{~cm}$

- thickness : $15 \mathrm{~mm} \mathrm{~Pb}$ from the floor to an height of 50 $\mathrm{cm}$. $10 \mathrm{~mm} \mathrm{~Pb}$ from $50 \mathrm{~cm}$ to $100 \mathrm{~cm}$. $5 \mathrm{~mm} \mathrm{~Pb}$ from $100 \mathrm{~cm}$ to $150 \mathrm{~cm}$.

- cover : $10 \mathrm{~mm}$ PMMA on the face of the barrier - total weight: $230 \mathrm{~kg}$ oriented towards the patient.

In order to absorb the Bremsstrahlung emerging from the patient during the treatment, moreover, "beam stoppers" mounted on casters and having the following characteristics were designed and built:

TABLE VII

Dose in air (expressed in $\mu \mathrm{G} / 100$ pulses) at different angles and for different lead thickness

\begin{tabular}{|c|c|c|c|c|c|c|c|}
\hline $\begin{array}{c}\text { Angle } \\
\text { Distance }(\mathrm{cm})\end{array}$ & $\begin{array}{c}0^{\circ} \\
130 \\
\end{array}$ & $\begin{array}{l}30^{\circ} \\
140\end{array}$ & $\begin{array}{l}45^{\circ} \\
150\end{array}$ & $\begin{array}{l}60^{\circ} \\
150\end{array}$ & $\begin{array}{l}90^{\circ} \\
130\end{array}$ & $\begin{array}{l}135^{\circ} \\
165 \\
\end{array}$ & $\begin{array}{l}180^{\circ} \\
300\end{array}$ \\
\hline$\underset{\downarrow}{\mathrm{mm}} \mathrm{Pb}$ & & & & $\begin{array}{c}100 \mathrm{p} \\
\downarrow\end{array}$ & & & \\
\hline 0 & 160 & 221 & 556 & 540 & 360 & 19 & 3.2 \\
\hline 5 & 95.5 & 12.6 & 6.6 & 2.5 & 1.2 & 0.2 & 0.1 \\
\hline 10 & 70.2 & 9.4 & 4.7 & 1.7 & 0.8 & & \\
\hline 15 & 59.2 & 6.4 & 3.5 & 1.2 & 0.6 & & \\
\hline 20 & 43.1 & 5.2 & 2.8 & 0.9 & 0.4 & & \\
\hline 25 & 34.5 & 4.2 & 2 & 0.8 & 0.2 & & \\
\hline 30 & 28.2 & 3.2 & 1.5 & 0.6 & & & \\
\hline 35 & 23.1 & 2.5 & 1.2 & 0.4 & & & \\
\hline 40 & 16.1 & 2.0 & 1.0 & 0.22 & & & \\
\hline 45 & 13.7 & 1.6 & 0.6 & & & & \\
\hline 50 & 11.5 & 1.1 & 0.2 & & & & \\
\hline 55 & 9.1 & 0,9 & & & & & \\
\hline 60 & 7.5 & 0.2 & & & & & \\
\hline 70 & 4.6 & & & & & & \\
\hline 80 & 2.5 & & & & & & \\
\hline 100 & 1.0 & & & & & & \\
\hline 120 & 0.4 & & & & & & \\
\hline 140 & 0.2 & & & & & & \\
\hline
\end{tabular}




\section{G. Tosi y M. Ciocca}

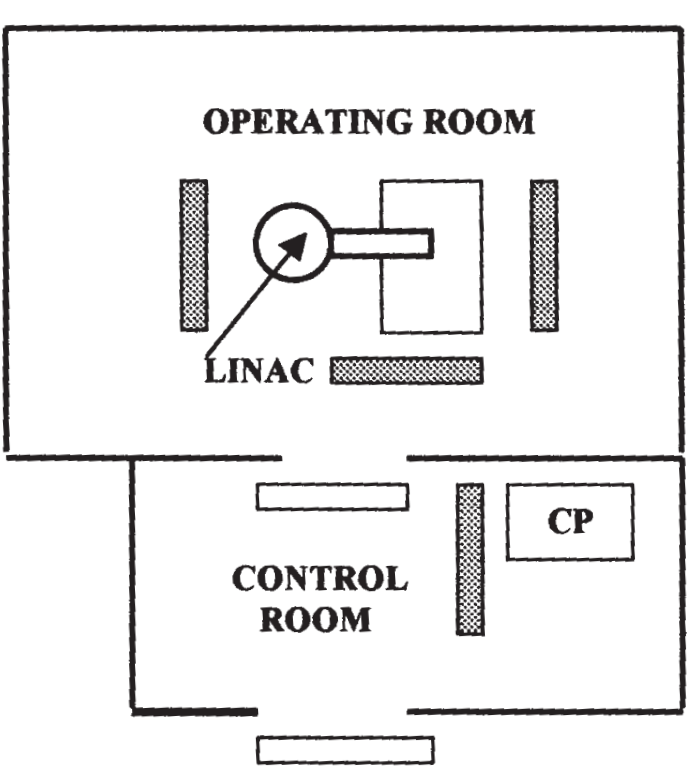

Fig. 7. Schematic layout of the operating room and of the control room, where the control panel (CP) of the linac is installed Clinical procedure.

$$
\begin{array}{ll}
\text { - width } & : 45 \mathrm{~cm} \\
\text { - length } & : 60 \mathrm{~cm} \\
\text { - thickness } & : 150 \mathrm{~mm} \mathrm{~Pb} \\
\text { - weight } & : 330 \mathrm{~kg}
\end{array}
$$

These beam stoppers are interlocked with the linacs, so as to make the emission of radiation impossible if the they are not placed under the operating table during the emission of the beam. A schematic layout of the operating room and of the control room, where the control panel (CP) of the linacs is installed is shown in Fig. 7.

After the surgeon has removed the tumour, a technician, assisted by a medical physicist, brings the linac nearby the operating table and helps the radiotherapist and the surgeon to put the applicator (that is hard-docked to the radiating head of the linac) in the treatment position (s. Fig. 8). In the meantime, a series of light- and acoustic warnings is activated; immediately after, the technician places the mobile barriers in the right positions around the operating table and all the personnel (surgeon, anaesthetist, radiotherapist, medical physicist, technician, nurses of the operating room, etc.) leaves the operating room and remains in the control room, from which the patient can constantly be controlled via the window of the door of the operating room and a telecamera. The radiotherapist prescribes the energy and the dose and the medical physicist starts the irradiation. The personnel classified as exposed worker due to its usual activities (radiotherapist, technician, medical physicist) wears its usual personal dosimeters.
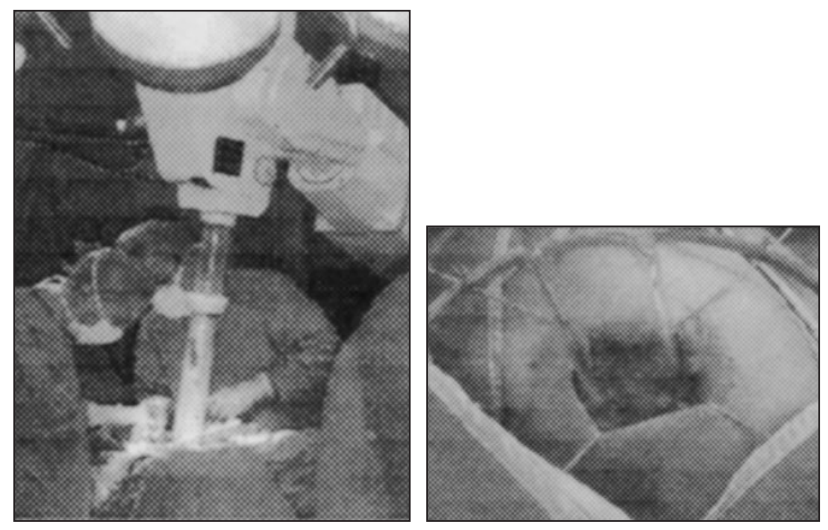

Fig. 8. Positioning of the applicator.

The other personnel, classified as not exposed (surgeon, anaesthetist, nurse, etc.) was monitored with a personal dosimeter only for the first three months, in order to check the adequacy of the classification. All the dosimeters indicated a value of the effective dose $<50 \mu \mathrm{Sv}$, thus confirming the adequacy both of the classification and of the barriers.

\section{Conclusions}

Up to april $30^{\text {th }}$ o 2004,889 early breast cancer patients and 25 other types of tumours have been treated, with very good results as regards both the long-term local control of the tumour and the absence of unwanted sideeffects.

In vivo dosimetry was also performed, using calibrated radiochromic films (s. Radiotherapy and Oncology 2003; 69:285-9) and/or Mosfet dosimeters. An agreement within $\pm 5 \%$ between the prescribed dose and the actually delivered dose was found.

As far as radiation protection is concerned, during the first month of activity, and afterwards with a quarterly frequency, environmental film and TLD-dosimeters were placed inside the adjacent, underlying and overlaying spaces; all the results were always very near to the natural background; even dosimeters placed on the inner face of the walls of the operating rooms revealed doses very low, $<6 \mathrm{mSv} / \mathrm{y}$.

During 5 years of employ of Novac 7 , only in 7 planned cases it was impossible to deliver the treatment due to faults of the equipment; a stop of nearly one month was necessary in 2003 for the change of the electron gun. After 3 months of employ, no fault has occurred with LIAC.

These results allow to conclude that the described mobile linacs are very reliable and can be sabely used for intraoperative radiotherapy. In early breast cancer patients this treatment technique, when indicated, can represent a valid alternative to post-surgical external radiotherapy. If adequate radiation protection measures are adopted, no risk exists for the involved personnel. 